\title{
Household Electrosmog
}

Wathiq Abdul-Razzaq, West Virginia University, USA

Ryan K. Bushey, West Virginia University, USA

\begin{abstract}
Electromagnetic wave energy is prevalent in the environment and is generated by numerous sources. This invisible electromagnetic wave/radiation pollution is termed Electrosmog. When exposed to this electromagnetic energy, health effects in humans have been observed and subsequent standards for emission have been set. A common household appliance that generates this energy is the microwave oven. This study evaluates the intensity of electromagnetic radiation leakage from microwave ovens subject to standard exposure levels and the possibility of biological effects at levels below the standards. We have taken the exposure levels into account in an effort to generate comparisons between microwave ovens according to their age. These comparisons have allowed us to create awareness of the possibility of harmful levels of electromagnetic radiation in our homes.
\end{abstract}

Keywords: Indoor Electrosmog; Radiation Leakage; Environmental Hazard

\section{INTRODUCTION}

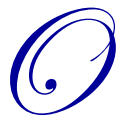

ne of the more recent challenges in the field of health is the increased electromagnetic radiation in the environment, also known as Electrosmog, which is generated by various household and commercial devices that require the use of the energy of electromagnetic waves. One such device that has been in use for several decades in our kitchens is the microwave oven which generates an electromagnetic wave with a frequency of $2.45 \mathrm{GHz}$. Consisting of multiple power levels, one must be careful of any radiation emitted from leaks within the surrounding area.

Standards have been set for the allowed leakage and exposure to various frequencies of waves in the Radio Frequency (RF) spectrum. The Institute of Electrical and Electronics Engineers (IEEE) has set maximum exposure levels in both controlled and uncontrolled environments for frequencies ranging from $3 \mathrm{kHz}$ to $300 \mathrm{GHz}$ (IEEE-SA Standards Board, 1999). Essentially, a controlled environment is determined to be an area in which the personnel are aware and may have control of the exposure, whereas an uncontrolled environment is an area where the personnel have no awareness of the exposure. Here, it is assumed that a microwave oven will be utilized in an uncontrolled environment. In this case, the maximum exposure level at the frequency of $2.45 \mathrm{GHz}$ is $1.633 \mathrm{milli}$ $\left(10^{-3}\right)$ Watt per centimeter squared or $\left(\mathrm{mW} / \mathrm{cm}^{2}\right)$ at $5 \mathrm{~cm}$ away from the source of the radiation, according to IEEE. The Food and Drug Administration (Code of Federal Regulations, 2006) has paid special attention to exposure levels emitted specifically from microwave ovens. They recommend a lower maximum exposure level than IEEE; namely, that at a distance of $5 \mathrm{~cm}$ from the source, a microwave oven will have a maximum leakage of 1.0 $\left(\mathrm{mW} / \mathrm{cm}^{2}\right)$ before customer purchase. However, the FDA suggests a much higher maximum exposure level of 5.0 $\mathrm{mW} / \mathrm{cm}^{2}$ at $5 \mathrm{~cm}$ after the oven has been purchased and used by the customer. Nonetheless, these maximum acceptable limits do not indicate that there are no biological effects at lower than these exposure levels of radiation.

Research into biological effects is described to be limited to mostly thermal effects that are based on heating the tissues during exposure to RF radiation. Kundi (2000) details how other factors may contribute to affecting biological processes aside from thermal effects. The research he cited suggests that various non-thermal biological effects possibly occur at the micro $\left(10^{-6}\right)$ Watt per centimeter squared or $\mu \mathrm{W} / \mathrm{cm}^{2}$ intensity range, which is on the order of one thousand times less than standards set forth by the IEEE and the FDA. While no fixed limit on biological effects can be concluded, we must still be aware of the possible consequences of electrosmog even at levels below the IEEE and FDA standards. 
Sage (2012) cited, in The BioInitiative 2012 Report, the work of many scientists who warned from the danger of RF energy even at low intensity exposure. The danger cited included loss of DNA repair capacity in human stem cells and carcinogenicity in humans. It was suggested in the report that existing public safety limits were inadequate to protect public health and that new safety limits were needed.

Since small intensities of RF radiation much lower than the FDA/IEEE standards can have the potential to be a health hazard, we conducted this study on RF radiation from microwave ovens.

\section{METHOD}

We chose to examine microwave ovens of different ages but of the same brand. Three ovens were chosen, manufactured in 1986, 1991, and 2007. Because of variations in power of these microwave ovens, we normalized the intensity to $1200 \mathrm{~W}$ in an effort to compare leaked intensities of the three ovens. For each dataset recorded, we took several measurements around the entirety of each microwave oven as a basis for our comparison. The device we used for measuring the intensity was AlphaLab's TriField meter (referenced below). We measured the intensity of the leaked radiation as a function of distance, where the intensity is measured in $\left(\mathrm{mW} / \mathrm{cm}^{2}\right)$ and the distance is recorded in increments of $5 \mathrm{~cm}$ from $0 \mathrm{~cm}$ to $100 \mathrm{~cm}$. Several points around the microwave ovens were measured for leaked intensity as a function of distance. That included a point on the right-hand side of the ovens near the top, as well as the lower right-hand corner of the door, the middle of the door on the bottom, the top of the oven near the back, the left-hand side of the oven near the top, and the left-hand side of the oven near the bottom. Note must be taken that the intensities measured were not completely stable at the measurements, but fluctuated periodically. This fluctuation may be partly due to the irregularity of the waves generated by the magnetron in the microwave oven and wave interference. The most frequently occurring value of the intensity, as well as the maximum readings of the intensity, were considered in our analysis.

The greatest leakage was seen to occur along the lower seal of the door for all ages of microwave ovens. The lower right-hand side (corner) of the door, close to the timer panel and the middle of the door on the bottom, emitted greater radiation intensities. We chose to show the data of these two points in Figures 1 and 2. Unfortunately, this area of greatest leakage is where most people stand due to the location of the timer panel and the door lock for opening and closing the door. It was observed (in the figures) that the oldest model emitted higher radiation intensity than recent ovens and the newest model had the lowest relative intensity at these points.

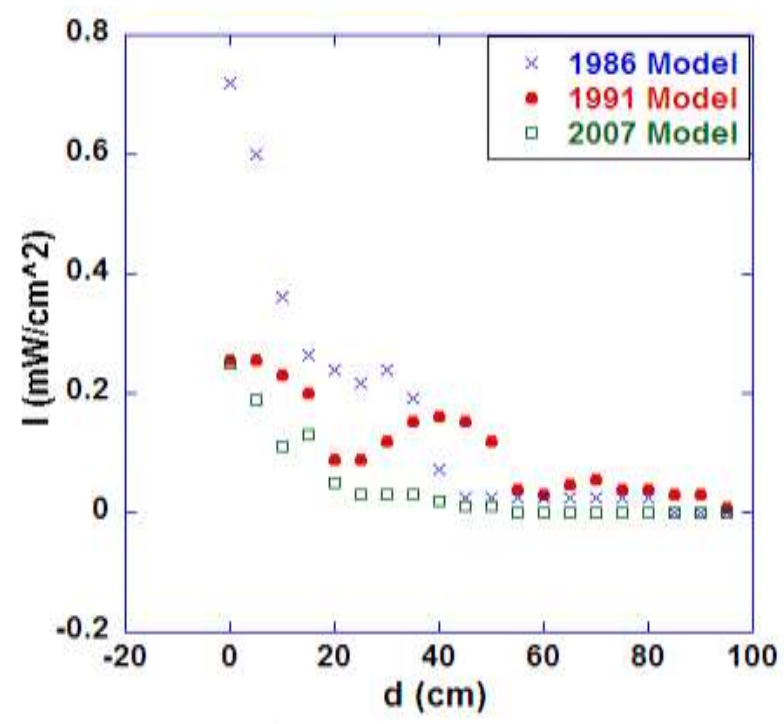

Figure 1: Normalized Intensity I At The Lower-Hand Corner Of The Door As A Function Of Distance d For Three Microwave Ovens Of The Same Brand But Manufactured In Different Years, 1986, 1991 And 2007 


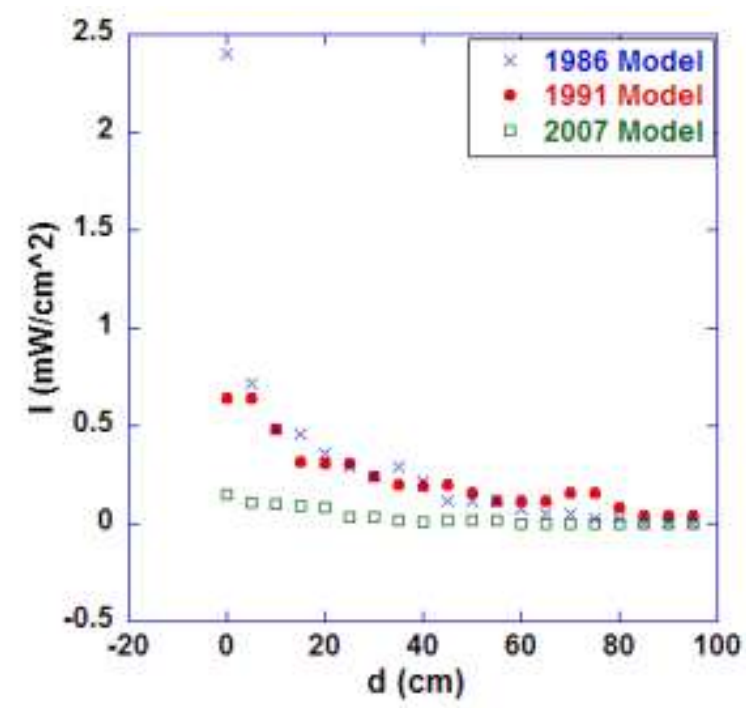

Figure 2: Normalized Intensity I At The Middle Of The Door On The Bottom As A Function Of Distance d For Three Microwave Ovens Of The Same Brand But Manufactured In Different Years, 1986, 1991 And 2007.

\section{RESULTS}

When evaluating the data we must consider the fluctuations in the data points and we paid particular attention to the non-normalized maximum values at the point along the lower right-hand corner of the door, as seen in Table 1, in units of $\mathrm{mW} / \mathrm{cm}^{2}$. We chose this point for evaluation as it had the greatest leakage intensity coupled with being closest to where people interact with the microwave oven.

Table 1: Maximum-Non-Normalized Intensity Values Along Lower Right Door Seal For Three Microwave Ovens Of The Same Brand But Different Manufacturing Dates

\begin{tabular}{|l|c|c|c|}
\hline \multicolumn{1}{|c|}{$\mathbf{d}(\mathbf{c m})$} & $\mathbf{I}_{\mathbf{m a x}}\left(\mathrm{mW} / \mathrm{cm}^{2}\right)$ & $\mathbf{I}_{\mathbf{m a x}}\left(\mathrm{mW} / \mathrm{cm}^{2}\right)$ & $\mathbf{I}_{\mathbf{m a x}}\left(\mathrm{mW} / \mathrm{cm}^{2}\right)$ \\
\hline 0 & $\mathbf{1 9 8 6}$ & $\mathbf{1 9 9 1}$ & 0.40 \\
\hline 5 & 1.00 & 0.32 & 0.25 \\
\hline 10 & 0.35 & 0.32 & 0.25 \\
\hline 15 & 0.35 & 0.29 & 0.20 \\
\hline 20 & 0.20 & 0.25 & 0.19 \\
\hline 25 & 0.20 & 0.11 & 0.15 \\
\hline 30 & 0.20 & 0.11 & 0.15 \\
\hline 35 & 0.15 & 0.15 & 0.08 \\
\hline 40 & 0.10 & 0.19 & 0.05 \\
\hline 45 & 0.15 & 0.20 & 0.00 \\
\hline 50 & 0.08 & 0.19 & 0.00 \\
\hline 55 & 0.06 & 0.15 & 0.00 \\
\hline 60 & 0.06 & 0.05 & 0.00 \\
\hline 65 & 0.04 & 0.04 & 0.00 \\
\hline 70 & 0.03 & 0.06 & 0.00 \\
\hline 75 & 0.03 & 0.07 & 0.00 \\
\hline 80 & 0.02 & 0.05 & 0.00 \\
\hline 85 & 0.02 & 0.05 & 0.00 \\
\hline 90 & 0.00 & 0.04 & 0.00 \\
\hline 95 & 0.00 & 0.04 & 0.00 \\
\hline 100 & 0.00 & 0.01 & \\
\hline
\end{tabular}


Quantitatively, the maximums were observed periodically over various time intervals depending on distance from the oven door. For example, the 1986 model most frequently measured $0.25 \mathrm{~mW} / \mathrm{cm}^{2}$ at $5 \mathrm{~cm}$ from the door, but periodically it fluctuated up to $0.35 \mathrm{~mW} / \mathrm{cm}^{2}$ approximately every 2 seconds. Other data points fluctuated to their maximums varying from 2 to 20 seconds. The fluctuations further depended on the microwave oven, as the 1991 model had very minimal measurable fluctuations over the distance measured.

When comparing these maximum values, we see that they fall within the approved safety guidelines that have been previously discussed. At a distance of $5 \mathrm{~cm}$, each oven is well within the strictest upper limit of 1.0 $\mathrm{mW} / \mathrm{cm}^{2}$ as characterized by the FDA. After normalization, we further observe the stable values within these recommended limits as well. However, we also note that the leaked radiation, especially along the bottom door seal, is significantly within the magnitude of tenths of milliwatts per square centimeter out to a distance of $50 \mathrm{~cm}$, which is quite larger than the suggested range by Kundi of microwatts per square centimeter within which he claims that biological effects may occur.

\section{CONCLUSION}

For all three microwave ovens we examined of same brand but different age, the measured leakage intensities seem to be greatest in front of the door along the bottom seal. As expected, age has a negative effect on leakage, probably due to either abuse or improved manufacturing methods when comparing microwave ovens made by the same company. Along the door seal on the bottom, we observe that the older microwave oven leaks much worse than the newer oven.

The data further suggests that all microwave ovens tested in this study are clearly within IEEE and FDA guidelines for safe RF radiation emission. As age increases, we see our normalized data indicate larger leakage intensity at near distances. Because of this fact, questions may arise if the microwave ovens are not replaced but are allowed to operate for many years, subject to IEEE and FDA limits.

However, if suggestions by Kundi about biological effects are followed, then our comparisons suggest that the leaked intensities of these microwave ovens are quite possibly harmful. By looking at the research on mice (Repacholi et al., 1997), Kundi derives a preliminary calculated level of exposure to be $0.117 \mu \mathrm{W} / \mathrm{cm}^{2}$ for the human public. When evaluating our data as discussed, we see that the exposure from microwave ovens is much greater than this value at significant distances from the source of the leak. Our intention is not to conclude that the microwave radiation is indeed harmful, but to raise awareness of this leaked radiation intensity and the possibility that it is unfavorable to health subject to preliminary research. The difficulty with making these comparisons is that reference levels for biological effects have yet to be decided upon conclusively in the scientific community due to many factors. With our everyday household and commercial appliances emitting this electrosmog, we suggest this be a focus for environmental research.

\section{AUTHOR INFORMATION}

Dr. Abdul-Razzaq received his $\mathrm{PhD}$ in 1986 from the University of Illinois at Chicago. He completed his postdoctorate work at Michigan State University. Currently he is Professor of Physics and Director of Introductory Physics Curriculum at West Virginia University. In recent years he has had research activities in diverse areas including studies of magnetic and transport properties of thin films and multilayers, studies of magnetic nanoparticles, and applied studies related to health and environment. In addition, he does research in education. E-mail: wabdulra@wvu.edu (Corresponding author)

Mr. Bushey received his Bachelor of Science in Physics from the University of Vermont in 2001, where his course work focus was in biophysics, specifically protein dynamics. Upon graduation he became a lead software engineer and then decided to pursue a graduate degree in order to become a high school physics teacher. He earned an M.S. degree in education from West Virginia University and currently is a physics teacher at Enosburg Falls High School, Vermont. E-mail: rbushey@enosburgk12.net 


\section{REFERENCES}

1. $\quad$ http://www.trifield.com/content/trifield-meter/

2. $\quad$ Food and Drug Administration (2006), Code of Federal Regulations 21 C.F.R. 1030.10.

3. IEEE-SA Standards Board. (1999). IEEE standard for safety levels with respect to human exposure to radio frequency electromagnetic fields, $3 \mathrm{kHz}$ to $300 \mathrm{GHz}$. New York: IEEE.

4. Kundi, M. (2000). Environmental health issues of radiofrequency and microwave exposure. http://www.salzburg.gv.at/Proceedings_(06)_Kundi.pdf

5. Sage, C (2012) The BioInitiative 2012 Report: www.bioinitiative.org

6. Repacholi M.H., Basten A., Gebski V., Noonan D., Finnie J., Harris A.W. (1997). Lymphomas in E $\mu$-Pim1 transgenic mice exposed to pulsed $900 \mathrm{MHz}$ electromagnetic fields. Radiation Research, 147, 631-640. 
NOTES 